\title{
Controller Design for Petri Net with Uncontrollable Transition
}

\author{
Ze Tao $^{1 *}$, Xiaoxia Liu ${ }^{2}$ \\ ${ }^{1}$ School of Mechanical Engineering, Shenyang Ligong University, Shenyang (110159), China \\ ${ }^{2}$ School of Electrical \& Mechanical Engineering, Henan University of Technology, Henan (450001), China \\ *Corresponding author's Email: taoze@tsinghua.edu.cn
}

\begin{abstract}
IA Petri net controller method for Petri net model with uncontrollable transitions that enforces the conjunctions a set of linear inequalities on the Parikh vector or place markings is proposed. The method for constructing a Petri net feedback controller is based on part design and Petri net reduction technique. Constraints are classified into admissible and inadmissible constraints. Matrix-Transformation method is proposed to transform the inadmissible constraint into admissible one to construct the controller. The method eases the design of controller and holds remarkable advantages especially for systems with large scale, because it only considers local incident matrix related to the place and Parikh constraints and uncontrollable transitions. Finally, the method is proved to be simple and efficient through some examples.
\end{abstract}

Keywords: Petri net controller; OR-logics; inhibitor arcs; uncontrollable transition

\section{Introduction}

In a flexible manufacturing system (FMS), different types of raw parts enter the system at discrete points of time and are processed concurrently, sharing a limited number of resources such as machine tools, automated guided vehicles, robots, buffers and fixtures. In such an FMS, each raw part follows a pre-established production sequence through the set of system resources. These production sequences are executed concurrently and therefore have to compete for the set of shared resources. This competition can cause deadlocks, in which each of a set of two or more jobs keeps waiting indefinitely for the other jobs in the set to release resources [1]. Deadlocks and related blocking phenomena often cause unnecessary productivity loss and therefore it is essential to develop a way to ensure that deadlocks never occur in an FMS. Petri nets have been taken as a tool for control synthesis of discrete event systems for their power on compact description of the state space, modeling of systems, graphical function, concurrency description of events and distributed state representation.

Mainly two types of Petri net (PN) controllers have been proposed in the literature [2]: the structural controller, where control policy is represented as a net structure, and the logical controller, where control actions (event enabling/disabling) are computed by an on-line controller as a feedback function of the marking of the system. For structural controller, many researchers have studied [3]. There are several advantages in structural controller compared with logical controller: computation of control action is faster, and the same PN execution algorithms may be used for both the original system and the supervisor. In addition, a closed-loop model of the system under control can be built with standard net composition constructions. The existence of liveness-ensuring supervisors for Petri nets with uncontrollable transitions has been studied in $[4,5]$. Techniques for deadlock avoidance have been proposed by a variety of researchers $[1,6$, 7]. These techniques involve analysis of the siphons 
or other similar structures within the Petri net plant. Often, the resulting controllers can be expressed by supervisors enforcing sets of linear inequalities on the reachable plant states. The main idea is that constraints transformation, but sometimes it will induce OR-logic linear constraint.

In this paper we propose a new approach to supervisory control of discrete event system (DES) for forbidden state problems, which couples supervisory control theory to the PN models with uncontrollable transitions through inhibitor arcs. Finally, compared with those methods in $[4,8,9]$ through examples, the method is proved to be simple and efficient and it can avoid deadlocks.

\section{Controller Design Based on Place Mark- ing}

Supervisory control of a DES is illustrated in Figure 1. The architecture consists of four parts: (1) the discrete event system (DES) to be controlled, (2) the controller (supervisor), (3) sensor readings, regarded as outputs from the DES and as inputs to the controller, and (4) control actions, regarded as outputs from the controller and as inputs to the DES. The supervisor must guarantee that no forbidden state will be reached, that specified target states remain reachable (non-blocking) and that controlled behavior is maximally permissive, i.e., the supervisor does not unnecessarily constrain system operation and is in this sense 'optimal'.

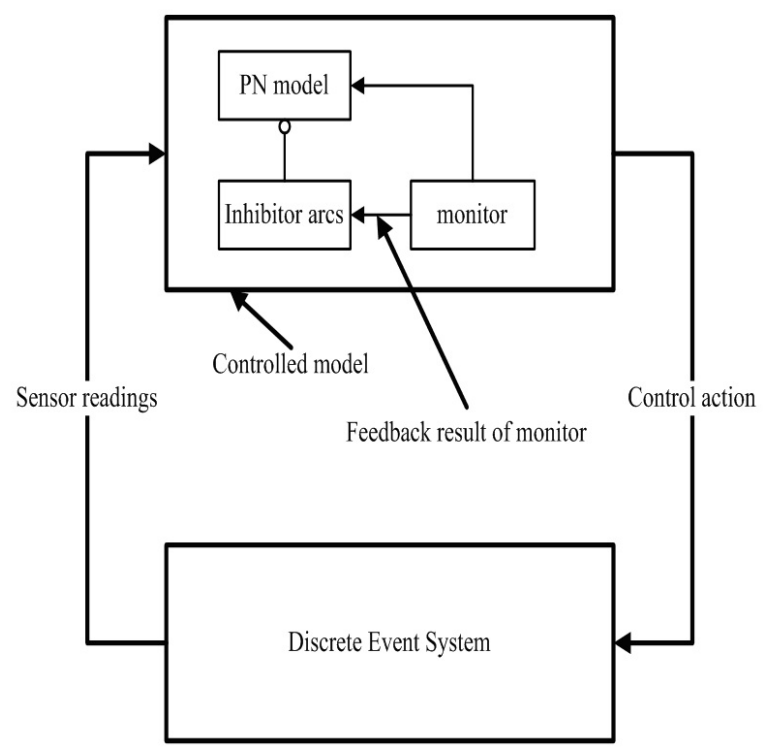

Figure 1 Supervisory control of a DES

The plant and the monitor are assumed to run concurrently as follows. The occurrence of an event in the plant is transmitted to the controlled model as a plant output through sensory feedback, resulting in the controlled model state change. The monitor functions and the inhibitor arcs as a state-feedback controller, whose enabling/disabling control actions are output as a plant input, closing the feedback loop. The controlled behavior of the plant will be the subset of uncontrolled.

\subsection{Petri net reduction technique and part net de- sign}

The Petri net reduction technique is one of the important techniques to reduce Petri net size. Its aim is to reduce the Petri net model scale and to maintain the consistency of the Petri net, for example, live, safe and bounded. The method proposed in this paper is based on Wang's [8] Petri net reduction technique. A Petri net with $n$ places and $m$ transitions is given. Suppose there are $k$ constrained places $p_{i}(1 \leq i \leq k)$ in the net, and fuse $k$ constrained places into one place $p_{e}$, whose marking is equal to the sum of the markings of the constrained places, therefore it eases the design of the controller. Furthermore, it only considers the direct or indirect transitions related to the constraints based on part net design. After these transformations, the local incident matrix dimension of constrained places will not become large as scale increasing of system. So the computation required to find the Petri net controller is quite simple.

\subsection{Controller design for fully controllable}

In DES Petri net model, the logical conjunction of separate linear constraints involves marking only, which has the following form:

$$
C=L u \leq b
$$

where $L \in Z^{n_{c} \times m}, u \in Z^{m}, b \in Z^{n_{c}}, m$ is the number of places, $n_{c}$ is the number of constraints, namely the number of controller. The detailed design of controller is as shown in Figure 2 when all transitions are controllable.

1). Find the local incident matrix of constrained place based on part net design and Petri net reduction technique.

$$
D=\stackrel{p_{1}}{\vdots} p_{k}\left[\begin{array}{cccc}
t_{1} & t_{2} & \cdots & t_{j} \\
d_{11} & d_{12} & \cdots & d_{1 j} \\
\vdots & \vdots & \vdots & \vdots \\
d_{k 1} & d_{k 2} & \cdots & d_{k j}
\end{array}\right]
$$

where $p_{1}, p_{2} \ldots p_{k}$ are constrained places, namely not all $l_{n_{c} j}$ are zero in corresponding matrix $L, t_{1}, t_{2} \ldots t_{j}$ 
are transitions related to the constrained places which can avoid the high dimension of Petri net model through only considering the direct or indirect transitions related to the constrained places.

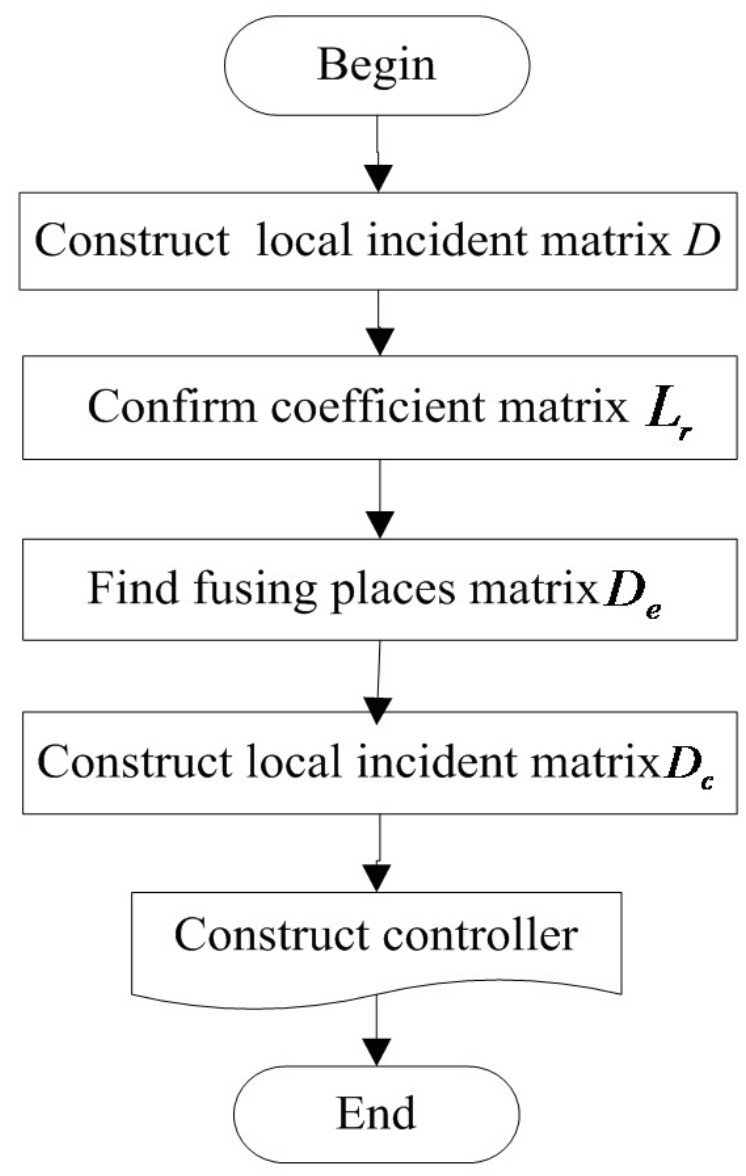

Figure 2 Flow chart of for fully controllable

2). Find the weight coefficient matrix of constrained places:

$$
L_{r}=\left[\begin{array}{cccc}
l_{11} & l_{12} & \cdots & l_{1 k} \\
\vdots & \vdots & \vdots & \vdots \\
l_{n_{c} 1} & l_{n_{c} 2} & \cdots & l_{n_{c} k}
\end{array}\right]
$$

where $k$ is the number of constrained places, the dimension of matrix $L_{r}$ is less than or equal to that of matrix $L_{r}$ since $L$ is simply the coefficient matrix of constrained places, but $L$ is the coefficient matrix of all places.

$3)$. Find fusing places matrix $D_{\varepsilon}$ [10]by Petri net reduction technique based on local incident matrix $D$ and coefficient matrix $L_{r}$ of constrained places, i.e.,

4). Find local incident matrix of controller places based on fusing places matrix $D_{\varepsilon}, D_{c}=D_{\varepsilon}$

Construct the controller based on matrix $D_{c}$, and its initial tokens should be:

$$
u_{c 0}=b-L_{r} u_{r 0}
$$

$u_{r 0}$ is the initial token of constrained places.

\subsection{Controller Design for Petri Net Model with Uncontrollable Transition}

\subsubsection{Uncontrollable Transitions}

A transition is called uncontrollable if the firing of that transition may not be inhibited by an external action. The freedom of an uncontrollable transition to fire is limited solely by the structure and state of the plant. It must not contain an arc from a controller place to the uncontrollable transition.

In order for a Petri net controller to inhibit a transition, it must contain an arc from a controller place to the transition. The transition will be disabled if the number of tokens in the control place is less than the arc weight. The controller is inadmissible if it contains an arc from a controller place to the uncontrollable transition.

\subsubsection{Controller Designing}

The detailed design of controller is as shown in Figure 3 when there are uncontrollable transitions in the Petri net model.

1). Find the local incident matrix of constrained place based on part net design and Petri net reduction technique. where $p_{1}, p_{2} \ldots p_{k}$ are constrained places, namely not all $l_{n_{c}, j}$ are zero in corresponding matrix $L, t_{1}, t_{2} \ldots t_{j}$ are transitions related to the constrained places which can avoid the high dimension of Petri net model through only considering the direct or indirect transitions related to the constrained places. Where $n \geq 0 h \geq 0, t_{j+1}, \ldots, t_{j+n}$ are uncontrollable transitions, $p_{m}, \ldots, p_{m+h}$ are unconstrained places related to uncontrollable transitions.

2). Find the weight coefficient matrix $L_{r}$ of constrained places and unconstrained places related to uncontrollable transitions:

$$
L_{r}=\left[\begin{array}{ccccccc}
l_{11} & l_{12} & \cdots & l_{1 k} & l_{1 m} & \cdots & l_{1(m+h)} \\
\vdots & \vdots & \vdots & \vdots & \vdots & \vdots & \vdots \\
l_{n 1} & l_{n 2} & \cdots & l_{n k} & l_{n m} & \cdots & l_{n(m+k)}
\end{array}\right]
$$

where $k$ is the number of constrained places, $h$ is the number of unconstrained places related to uncontrollable transitions, the dimension of matrix $L_{r}$ is less than or equal to that of matrix $L$ since $L_{r}$ is simply the coefficient matrix of constrained places, but is the coefficient matrix of all places.

3). Admissible Constraint and Constraint Transformations

[1] Examine if the constraints are admissible. The constraints are admissible if $L_{r} D_{u c} \leq 0$ is true $\left(D_{u c}\right.$ 


$$
\begin{aligned}
& D_{e}=L_{r} D=\begin{array}{c}
p_{e 1} \\
\vdots
\end{array}\left[\begin{array}{cccc}
\sum_{e n_{c}}^{t_{1}} & t_{2} & \cdots & { }^{t_{j}} \\
\sum_{i=1}^{k} l_{1 i} d_{i 1} & \sum_{i=1}^{k} l_{1 i} d_{i 2} & \cdots & \sum_{i=1}^{k} l_{1 i} d_{i j} \\
\vdots & \vdots & \vdots & \vdots \\
\sum_{i=1}^{k} d_{i 1} & \sum_{i=1}^{k} l_{n_{c} i} d_{i 2} & \cdots & \sum_{i=1}^{k} l_{n_{c} i} d_{i j}
\end{array}\right] \\
& D_{c}=\quad \vdots\left[\begin{array}{cccc}
{ }^{t_{1}} & { }^{t_{2}} & \cdots & { }^{t_{j}} \\
-\sum_{i=1}^{k} l_{1 i} d_{i 1} & -\sum_{i=1}^{k} l_{1 i} d_{i 2} & \cdots & -\sum_{i=1}^{k} l_{1 i} d_{i j} \\
\vdots & \vdots & \vdots & \vdots \\
-\sum_{i=1}^{k} l_{n_{c} i} d_{i 1} & -\sum_{i=1}^{k} l_{n_{c} i} d_{i 2} & \cdots & -\sum_{i=1}^{k} l_{n_{c} i} d_{i j}
\end{array}\right]
\end{aligned}
$$

is the incident matrix corresponding to the uncontrollable transitions in matrix $D$ ), then go to 4).

[2] The constraints are inadmissible if $L_{r} D_{u c} \geq 0$, namely, it contains an arc from a controller place to the uncontrollable transition. Transform constraints $C$ to admissible constraints $C^{\prime}=L u \leq b$, causing $L_{r}^{\prime} D_{u c} \leq$ 0 through constraints transformation.

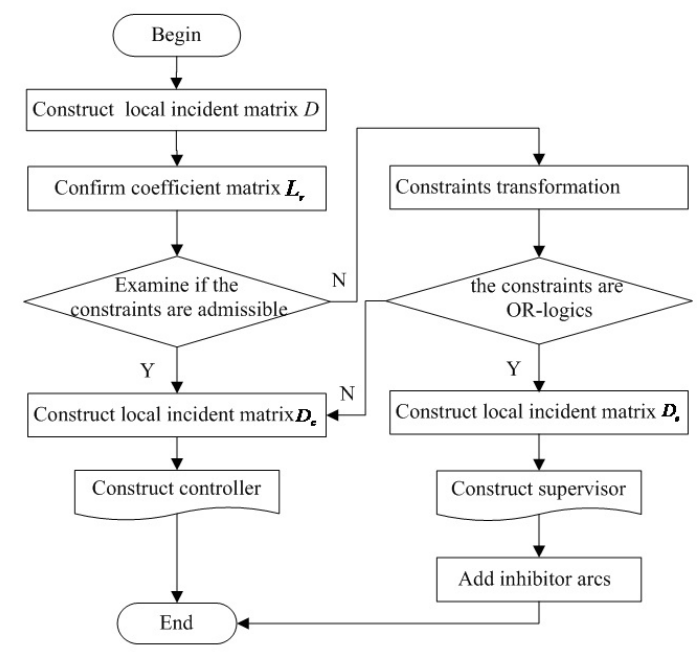

Figure 3 The flow chart of controller design

Theorem 1 Let $R \in Z^{n_{c} \times(k+h)}$, satisfy $R u_{r} \geq 0, \forall u_{r}$ ( $u_{r}$ are markings of constrained places); if $L_{r}^{\prime} u_{r} \leq b$, where $L_{r}^{\prime}=R+L_{r}$, then $L_{r} u_{r} \leq b$.

Proof: $L_{r}^{\prime} u_{r} \leq b$, then $\left(R+L_{r}\right) u_{r} \leq b, R u_{r}+L_{r} u_{r} \leq b$, substituting $R u_{r} \geq 0$ into $R u_{r}+L_{r} u_{r} \leq b$ gives.

In Theorem $1, L_{r}^{\prime}$ must satisfy $L_{r}^{\prime} D_{u c} \leq 0$. Find $L_{r}^{\prime}$ by matrix transformation, the detailed process can be stated as follows:

Let

$$
M=\left[\begin{array}{cc}
D_{u c} & I \\
L_{r} D_{u c} & 0
\end{array}\right]
$$

where $M \in Z^{\left(k+h+n_{c}\right) \times\left(n_{u c}+k+h\right)}, L_{r} \in Z^{n_{c} \times(k+h)}, D_{u c} \in$ $Z^{(k+h) \times n_{u c}}, I \in Z^{(k+h) \times(k+h)}, 0 \in Z^{n_{c} \times(k+h)} . \quad M(i, j)$ denotes the $(i, j)^{\text {th }}$ element of the matrix $M$, and let $j=1$.

(1) If $M(p, j)>0$ is true in the $M(h+h+1 \ldots k+$ $\left.h+n_{c}, j\right)$, then go to (2); otherwise $j=j+1$, carry out (1) again.

(2) If $M(q, j)<0$ is not true in the $M(1 \ldots k+h, j)$, then the legal controller cannot be designed firsthand as it needs transforming, otherwise find out $\min (\mid M(k+$ $\left.\left.h+1 \ldots k+h+n_{c}, j\right) \mid\right)$, which satisfies $M(q, j)<0$, then go to (3).

(3) If $|M(q, j)| \geq M(p, j)$ is true, then go to (a), otherwise go to (b):

(a) $M(p, \bullet)=M(p, \bullet)+M(q, \bullet)$ :

(b) $d=\operatorname{floor}(M(p, j) /|M(q, j)|)$,

If $\bmod (M(p, j), M(q, j))=0$,

Then $M(p, \bullet)=M(p, \bullet)+d M(q, \bullet)$;

Otherwise $M(p, \bullet)=M(p, \bullet)+(d+1) M(q, \bullet)$.

(4) Carry out (1) again until $M(p, j)>0$ is not true in $M\left(k+h+1 \ldots k+h+n_{c}, j\right)$, and now matrix $M$ is transformed into matrix $M^{\prime}$, so $L_{r}^{\prime}=R+L_{r}$ can be found. 


$$
D=\begin{gathered}
p_{1} \\
\vdots \\
p_{k} \\
p_{m} \\
\vdots \\
p_{m+h}
\end{gathered}\left[\begin{array}{ccccccc}
t_{1} & t_{2} & \cdots & t_{j} & t_{j+1} & \cdots & t_{j+n} \\
d_{11} & d_{12} & \cdots & d_{1 j} & d_{1(j+1)} & \cdots & d_{1(j+n)} \\
\vdots & \vdots & \vdots & \vdots & \vdots & \vdots & \vdots \\
d_{k 1} & d_{k 2} & \cdots & d_{k j} & d_{k(j+1)} & \cdots & d_{k(j+n)} \\
d_{m 1} & d_{m 2} & \cdots & d_{m j} & d_{m(j+1)} & \cdots & d_{m(j+n)} \\
\vdots & \vdots & \vdots & \vdots & \vdots & \vdots & \vdots \\
d_{(m+h) 1} & d_{(m+h) 2} & \cdots & d_{(m+h) j} & d_{(m+h)(j+1)} & \cdots & d_{(m+h)(j+n)}
\end{array}\right]
$$

Where

$$
M^{\prime}=\left[\begin{array}{cc}
D_{u c} & I \\
L_{r}^{\prime} D_{u c} & R
\end{array}\right]
$$

The new legal controller can be designed according to the new constraints $L_{r}^{\prime} u_{r} \leq b$, and it satisfies the rule of uncontrollable transition.

[3] After matrix transformation, the constraints are OR-logics linear constraints, then construct monitor according to 4) and 6).

4). Find fusing places matrix $D_{e}[6]$ by Petri net reduction technique based on local incident matrix $D$ and coefficient matrix $L_{r}$ of constrained places, i.e.,

5). Find local incident matrix of controller places based on fusing places matrix $D_{e}, D_{c}=-D_{e}$

Construct the controller based on matrix , and its initial tokens should be:

$$
u_{c 0}=b-L_{r} u_{r 0}\left(\text { or } u_{c 0}=b-L_{r}^{\prime} u_{r 0}\right)
$$

$u_{r 0}$ is the initial token of constrained places.

6). If the constraints are OR-logics linear constraints, then construct the monitor according to matrix $D_{e}$, and add inhibitor arcs from monitor to the transitions where the element in the matrix $D_{c}$ correspondence is $>0$, and $u_{c 0}=L_{r} u_{r 0}$.

\section{Controller Design Based on Parikh vector}

\subsection{Parikh Vector Constraints}

In DES Petri net model, the logical conjunction of separate linear constraints involves Parikh vector only, which has the following form:

$$
c v<b
$$

where $C \in Z^{m_{c} \times n}, b \in Z^{+m_{c}}, Z$ is the set of integers, $Z^{+}$is the set of non-negative, $\mathrm{n}$ is the number of transitions in the plant, $n$ is the number of places, $m_{c}$ is the number of constraints.

According to [5], for the linear inequality constraint (12), each place can be described with an inequality.
Consider the place $p_{1}$ in Figure 1: when transition $t_{1}$ fires once, then decreasing one token in place $p_{1}$. When transition $t_{2}$ fires once, then one token in place $p_{1}$ is decreased. Since gaining is greater than losing, the inequality is obtained:

$$
3+v_{2} \geq v_{1}
$$

formalizing:

$$
v_{1}-v_{2} \leq 3
$$

The places $p_{2}, p_{3}, p_{4}, p_{5}$ in the Figure 4 can be represented respectively:

$$
\begin{aligned}
& v_{3}-v_{1}-v_{4} \leq 0 \\
& v_{2}+v_{6}-v_{3} \leq 1 \\
& v_{4}-v_{5} \leq 0 \\
& v_{5}-v_{6} \leq 0
\end{aligned}
$$

If the constraints can be described with inequality (12), then the controller place can be obtained correspondingly to each constraint. The incidence matrix and initial markings of controller place is:

$$
D_{p c}=-c ; \mu_{c}=b
$$

\subsection{Controller Design for Partially Controllable}

In linear inequalities (12), if the transitions related to the Parikh vectors are uncontrollable, then the method taken is shown as follows:

Examine if the constraints are admissible. A set of constraints is admissible if $C_{u c} \leq 0$ is true, in spite of the inability to detect or control certain transitions. ( $C_{u c}$ is the coefficient matrix corresponding to the uncontrollable transitions in coefficient matrix $C$ ). Then construct the controller; otherwise, carry through constraints transformation.

Theorem 2: Let $R \in Z^{m_{c} \times m_{u c}}$ ( $m_{u c}$ is number of places related to uncontrollable transitions), $C^{\prime}=R D+C$, 


$$
\begin{aligned}
& D_{e}=L_{r} D=\quad \vdots\left[\begin{array}{ccccc}
t_{1} & \cdots & t_{j} & \cdots & t^{t_{j+n}} \\
\sum_{e n_{C}}^{k} l_{1 i} d_{i 1} & \cdots & \sum_{i=1}^{k} l_{1 i} d_{i j} & \cdots & \sum_{i=1}^{k} l_{1 i} d_{i(j+n)} \\
\vdots & \vdots & \vdots & \vdots & \\
\sum_{i=1}^{k} l_{n_{c} i} d_{i 1} & \cdots & \sum_{i=1}^{k} l_{1 i} d_{i j} & \cdots & \sum_{i=1}^{k} l_{n_{c} i} d_{i(j+n)}
\end{array}\right]
\end{aligned}
$$

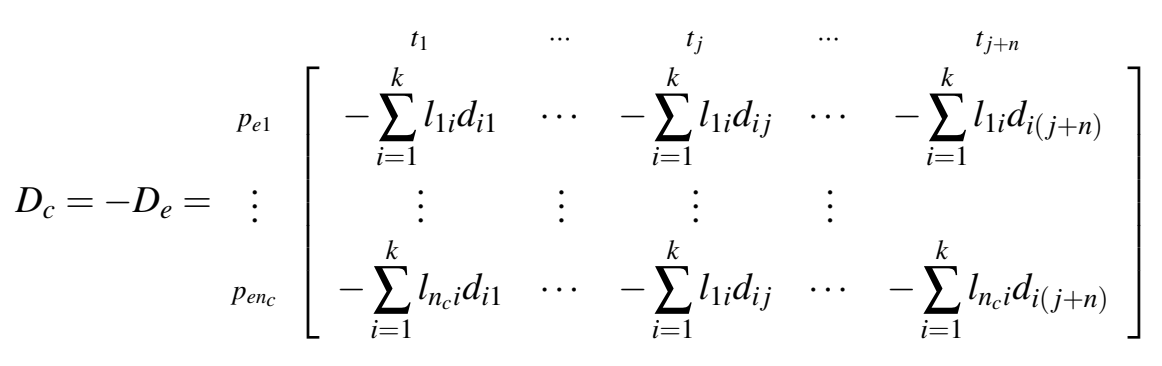

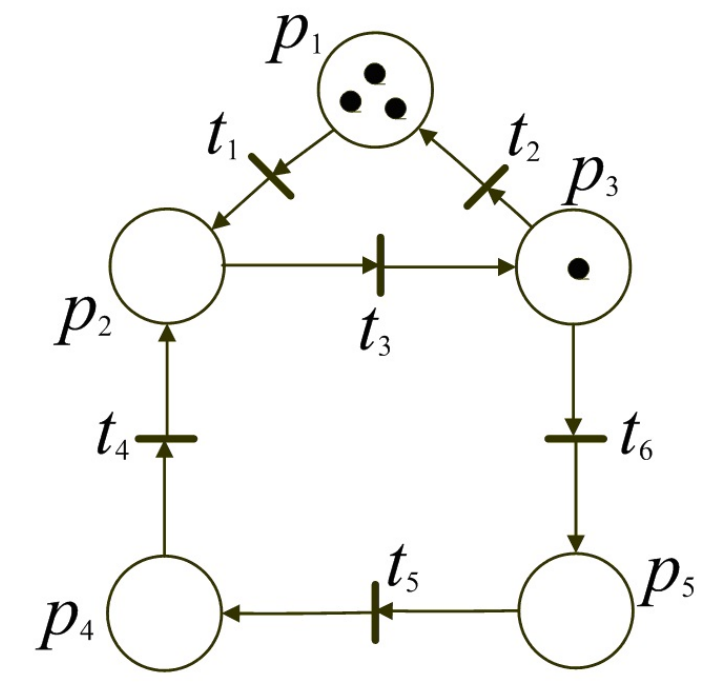

Figure 4 Petri net model

$b^{\prime}=b-R \mu_{0}$, where $R \geq 0, b^{\prime} \geq 0$, if $C^{\prime} v \leq b^{\prime}$ is true, then $C v \leq b$ is true.

Proof: $C^{\prime} v \leq b^{\prime}$, namely $(R D+C) v \leq b-R \mu_{0}$, then $R\left(D v+\mu_{0}\right)+C v \leq b$, since $\mu=D v+\mu_{0} \geq 0$, therefore $C v \leq b$.

Theorem 3: Let $R \in Z^{m_{c} \times m_{u c}}$, if

$$
\left\{\begin{array}{l}
R \geq 0 \\
b-R \mu_{0} \geq 0 \\
R D_{u c}+C_{u c} \leq 0
\end{array}\right.
$$

Let $C^{\prime}=R D+C, b^{\prime}=b-R \mu_{0}$, then $C^{\prime} v \leq b^{\prime}$ is a set of admissible constraints.

Proof: $C_{u c}^{\prime}=R D_{u c}+C_{u c} \leq 0$, namely the quality of uncontrollable transitions is satisfied for the Parikh vector constraints $\left(C^{\prime}, b^{\prime}\right)$, so $C^{\prime} v \leq b^{\prime}$ is a set of admissible constraints.

The method of transforming inadmissible constraints into admissible constraints is given according to theorem 2 and theorem 3.

$R$ in theorem 3 can be obtained according to matrix transformation:

Let

$$
\left[\begin{array}{ccc}
D_{u c} & \mu_{0} & I \\
C_{u c} & -b & 0
\end{array}\right]
$$

where $M \in Z^{\left(m_{u c}+m_{c}\right) \times\left(n_{u c}+m_{c}\right)}, D_{u c} \in Z^{m_{u c} \times n_{n c}}, C_{u c} \in$ $Z^{m_{c} \times n_{u c}}, I \in Z^{m_{u c} \times m_{u c}}, 0 \in Z^{m_{c} \times m_{u c}}$.

$M(i, j)$ denotes the $(i, j)^{t h}$ element of the matrix $M$, and let $j=1$.

(1) If $M(i, j)>0$ in the $M\left(m_{u c}+1 \ldots m_{u c}+m_{c}, j\right)$, then go to (2); otherwise $j=j+1$, carry out (1) again.

(2) If $M(q, j)<0$ is not true in the $M\left(1 \ldots m_{u c}, j\right)$, then the admissible controller cannot be designed, otherwise find out $\min \left(\left|M\left(m_{u c+1} \ldots m_{u c}+m_{c}, j\right)\right|\right)$ that satisfies $M(q, j)<0$, then go to (3).

(3) If $|M(q, j)| \geq M(p, j)$ is true, then go to (a), otherwise go to (b):

$$
\begin{aligned}
& \text { (a) } M(p, \bullet)=M(p, \bullet)+M(q, \bullet) \text {; } \\
& \text { (b) } \operatorname{floor}(M(p, j) /|M(q, j)|) \text {, } \\
& \text { If } \bmod (M(p, j), M(q, j))=0 \\
& \text { Then } M(p, \bullet)==M(p, \bullet)+d M(q, \bullet) \text {; } \\
& \text { Otherwise } M(p, \bullet)==M(p, \bullet)+(d+1) M(q, \bullet) \text {; }
\end{aligned}
$$


(4) Carry out (1) again until $M(p, j)>0$ is not true in $M\left(m_{u c}+1 \ldots m_{u c}+m_{c}, j\right.$; and now matrix $M$ is transformed into matrix $M^{\prime}=\left[\begin{array}{ccc}D_{u c} & \mu_{0} & I \\ C_{u c}^{\prime} & R \mu_{0}-b & R\end{array}\right]$, so $C^{\prime}=R D+C$ can be found.

The new admissible controller can by designed according to the new constraints: $C^{\prime} v \leq b^{\prime}$. Since it only considers the places related to uncontrollable transitions and the transitions related to above places, so it reduces the matrix dimension. The superiority is more remarkable especially for systems with large scale.

\section{Case Study}

\subsection{Case 1}

A simple Petri net is shown in Figure 5 (real line part) where $t_{3}$ and $t_{6}$ are uncontrollable transitions.

Suppose it is needed to design a controller to force the following combined constraints to be satisfied:

$$
u_{5}+u_{6} \leq 1
$$

The broken line part in Figure 3 is controller, which is designed with the method in this paper.

$$
\begin{gathered}
D_{e}=L_{r} D=\begin{array}{c}
p_{3} \\
p_{4} \\
p_{5} \\
p_{6} \\
p_{7}
\end{array}\left[\begin{array}{ccccccc}
t_{1} & t_{2} & t_{3} & t_{4} & t_{5} & t_{6} \\
1 & 0 & -1 & 0 & 0 & 0 \\
0 & 1 & -1 & 0 & 0 & 0 \\
0 & 0 & 1 & -1 & 0 & 0 \\
0 & 0 & 0 & 0 & -1 & 1 \\
0 & 0 & 0 & 0 & 1 & -1
\end{array}\right] \\
{\left[\begin{array}{lllll}
0 & 0 & 1 & 1 & 0
\end{array}\right]}
\end{gathered}
$$

From matrix $D$, the matrix $D_{u} c$ is:

$$
D_{u c}\left[\begin{array}{cc}
-1 & 0 \\
-1 & 0 \\
1 & 0 \\
0 & 1 \\
0 & -1
\end{array}\right] \quad L_{r} D_{u c}=\left[\begin{array}{ll}
1 & 1
\end{array}\right]
$$

Positive number is concluded in the matrix $L_{r} D_{u c}$, namely it contains an arc from the controller place to the uncontrollable transition, so inequality (13) is inadmissible, and it needs to be transformed.

From the constraint transformation method in 3), substitute $L_{r}$ and $D_{u c}$ into $M$, and in terms of constraint transformation method (1) to (4)

$$
\begin{aligned}
& R_{1}\left[\begin{array}{lllll}
1 & 0 & 0 & 0 & 1
\end{array}\right], \\
& R_{1}^{\prime}\left[\begin{array}{lllll}
0 & 1 & 0 & 0 & 1
\end{array}\right], \\
& L_{r}^{\prime}=R+L_{R}=\left[\begin{array}{lllll}
1 & 0 & 1 & 1 & 1
\end{array}\right] \text { or } \\
& L_{r}^{\prime}=\left[\begin{array}{lllll}
0 & 1 & 1 & 1 & 1
\end{array}\right]
\end{aligned}
$$

the new constraints are then:

$$
u_{5}+u_{6} \leq 1 \Rightarrow u_{3}+u_{5}+u_{6}+u_{7} \leq 1
$$

or

$$
u_{4}+u_{5}+u_{6}+u_{7} \leq 1
$$

The constraints are OR-logics linear constraints, while the controller cannot be designed with the method in $[8,11]$. According to the method in this paper, construct $D_{e}$,

$$
\begin{aligned}
& D_{e}=L-r D=\left[\begin{array}{llllll}
0 & 0 & 1 & -1 & -1 & 1
\end{array}\right] \\
& u_{c 0}=L_{r} u_{r 0}=0
\end{aligned}
$$

If take the method of Moody [9] to design the controller, then

$$
\begin{aligned}
& L= {\left[\begin{array}{ccccccc}
0 & 0 & 0 & 0 & 1 & 1 & 0
\end{array}\right] ; } \\
& D_{p}=\left[\begin{array}{cccccc}
-1 & 0 & 0 & 1 & 0 & 0 \\
0 & -1 & 0 & 1 & 0 & 0 \\
1 & 0 & -1 & 0 & 0 & 0 \\
0 & 1 & -1 & 0 & 0 & 0 \\
0 & 0 & 1 & -1 & 0 & 0 \\
0 & 0 & 0 & 0 & -1 & 1 \\
0 & 0 & 0 & 0 & 1 & -1
\end{array}\right] ;
\end{aligned}
$$

The dimension of matrix $L$ and matrix $D_{p}$ is much obviously higher than that of matrix $L_{r}$ and matrix $D$. Especially for systems with large scale, the computation complexity will increase as the dimension of matrix $L$ and matrix $D_{p}$ increase. But apply the method proposed in this paper, the dimension of matrix $L_{r}$ and matrix $D$ will not increase as the plant scale becomes large, so the method is efficient.

\subsection{Case 2}

Consider the typical example "cat and mouse" in DES (Figure 6). Figure 7a and $b$ are Petri net models for cat and mouse respectively, where transition c8 and transition $\mathrm{m} 5$ are uncontrollable [12]. It is necessary to design controllers to insure that cat and mouse are not in the same room at the same time. Describe the control target with place marking constraints in [13], however in [9], describe the constraints with Parikh vector.

It is needed to design a controller to force the following combined constraints to be satisfied:

$$
\left(v_{c 3}+v_{c 6}-v_{c 1}-v_{c 4}\right)+\left(v_{m 3}+v_{m 6}-v_{m 1}-v_{m 4}\right) \leq 1
$$




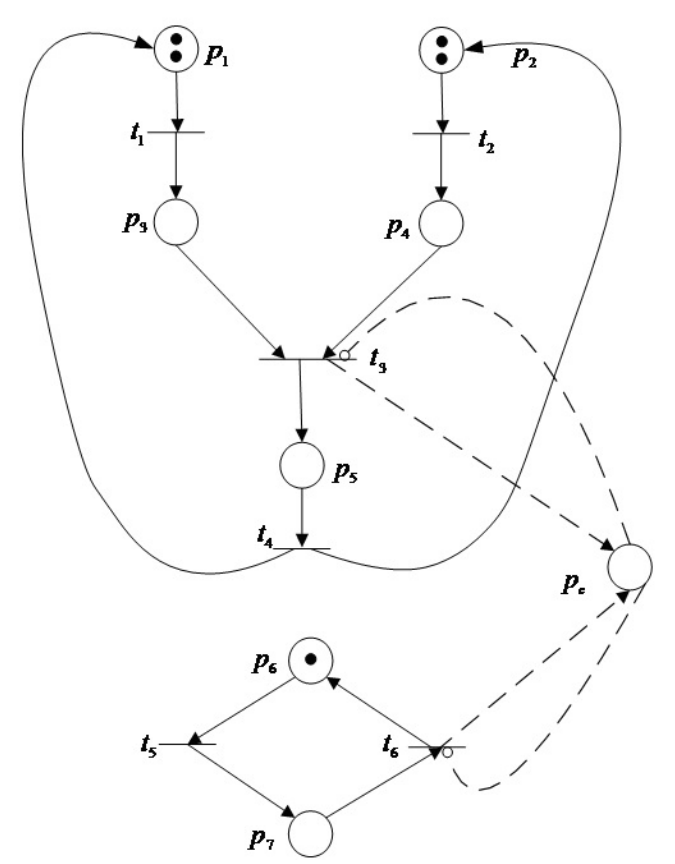

Figure 5 Petri net model

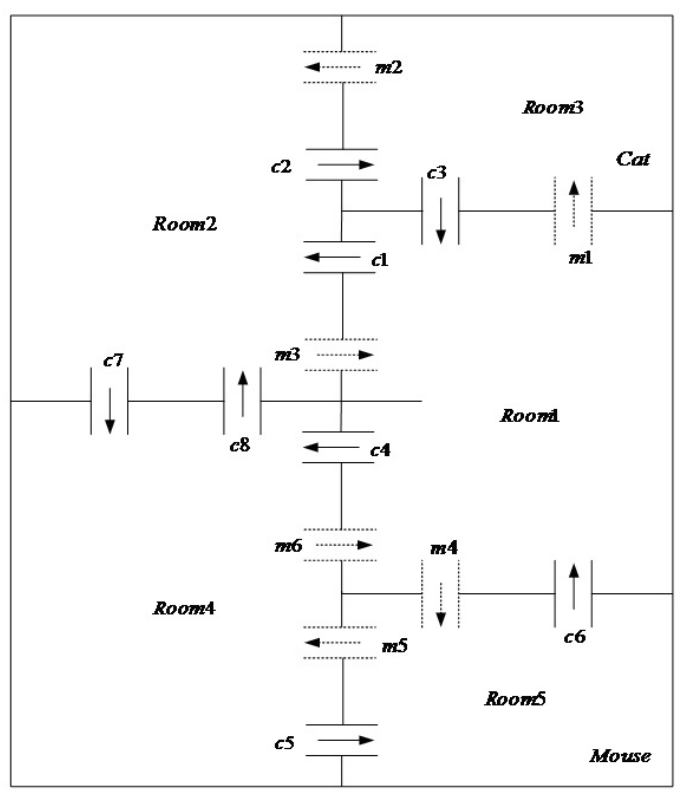

Figure 6 A controlled Petri net

$$
\begin{aligned}
& \left(v_{c 1}+v_{c 8}-v_{c 2}-v_{c 7}\right)+\left(v_{m 2}-v_{m 3}\right) \leq 1 \\
& \left(v_{c 2}-v_{c 3}\right)+\left(v_{m 1}-v_{m 2}\right) \leq 1
\end{aligned}
$$

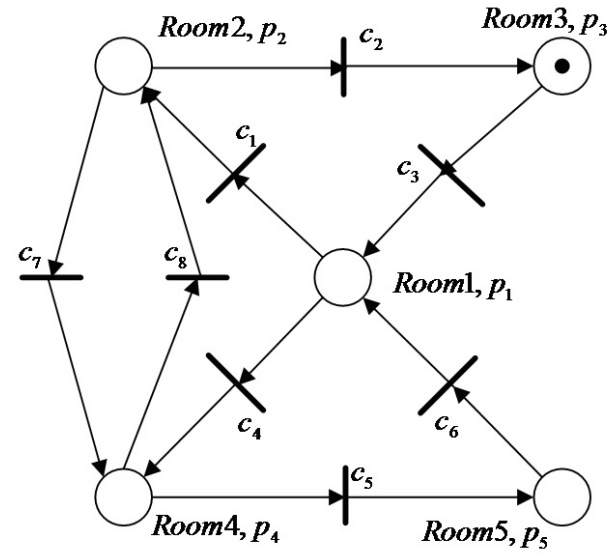

Figure 7 Model of "cat and mouse"

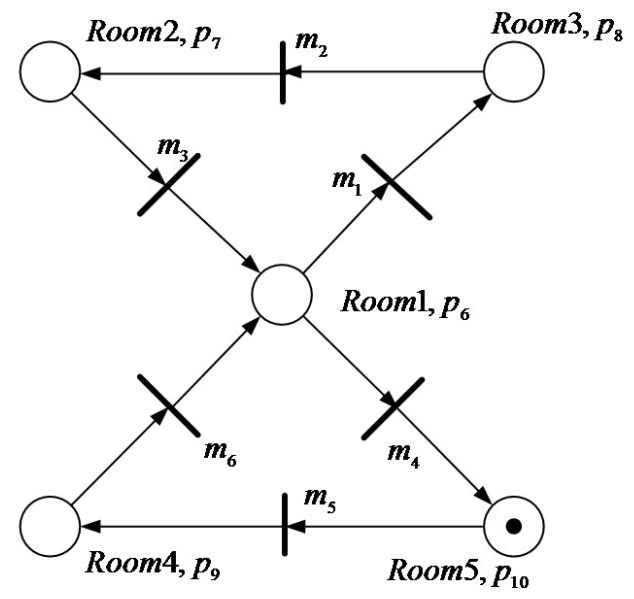

Figure 8 Petri net model of mouse

$$
\begin{aligned}
& \left(v_{c 4}+v_{c 7}-v_{c 5}-v_{c 8}\right)+\left(v_{m 5}-v_{m 6}\right) \leq 1 \\
& \left(v_{c 5}-v_{c 6}\right)+\left(v_{m 4}-v_{m 5}\right) \leq 1
\end{aligned}
$$

(17) and (19) are inadmissible constraints, due to the coefficients of uncontrollable transition $c_{8}$ and $m_{5}$. So, in this paper, it only considers constraints (17) and (19) to construct the controllers.

Construct local incident matrix $D$ which is local the incident matrix of places $p_{2}, p_{4}, p_{9}$ and $p_{10}$ that are related to uncontrollable transition $c_{8}$ and $m_{5}$. The transitions in matrix $D$ are related to places $p_{2}, p_{4}, p_{9}$ and $p_{10}$ and the transitions where the coefficients are not equal to 0 in constraints (17) and (19).

$$
D_{u c}=\left[\begin{array}{cc}
1 & 0 \\
-1 & 0 \\
0 & 1 \\
0 & -1
\end{array}\right]
$$




$$
D=\begin{gathered}
p_{2} \\
p_{4} \\
p_{9} \\
p_{10}
\end{gathered}\left[\begin{array}{ccccccccccc}
1 & -1 & 0 & 0 & 1 & 1 & 0 & 0 & 0 & 0 & 0 \\
0 & 0 & 1 & -1 & 1 & -1 & 0 & 0 & 0 & 0 & 0 \\
0 & 0 & 0 & 0 & 0 & 0 & 0 & 0 & 0 & 1 & -1 \\
0 & 0 & 0 & 0 & 0 & 0 & 0 & 0 & 1 & -1 & 0
\end{array}\right]
$$

The coefficient matrix corresponding to inadmissi-

ble constraints (11) and (13) is $C$ :

$$
C=\left[\begin{array}{ccccccccccc}
1 & -1 & 0 & 0 & -1 & 1 & 1 & -1 & 0 & 0 & 0 \\
0 & 0 & 1 & -1 & 1 & -1 & 0 & 0 & 0 & 1 & -1
\end{array}\right]
$$

where the coefficient matrix corresponding to uncontrollable transition $c_{8}$ and $m_{5}$ is:

$$
D_{u c}=\left[\begin{array}{cc}
1 & 0 \\
-1 & 1
\end{array}\right]
$$

Design the controllers with the method proposed in this paper:

$$
M=\left[\begin{array}{ccc}
D_{u c} & \mu_{0} & I \\
C_{u c} & -b & 0
\end{array}\right]=\left[\begin{array}{ccccccc}
1 & 0 & 0 & 1 & 0 & 0 & 0 \\
-1 & 0 & 0 & 0 & 1 & 0 & 0 \\
0 & 1 & 0 & 0 & 0 & 1 & 0 \\
0 & -1 & 1 & 0 & 0 & 0 & 1 \\
1 & 0 & -1 & 0 & 0 & 0 & 0 \\
-1 & 1 & -1 & 0 & 0 & 0 & 0
\end{array}\right]
$$

Obtain $M^{\prime}$ through matrix transformation:

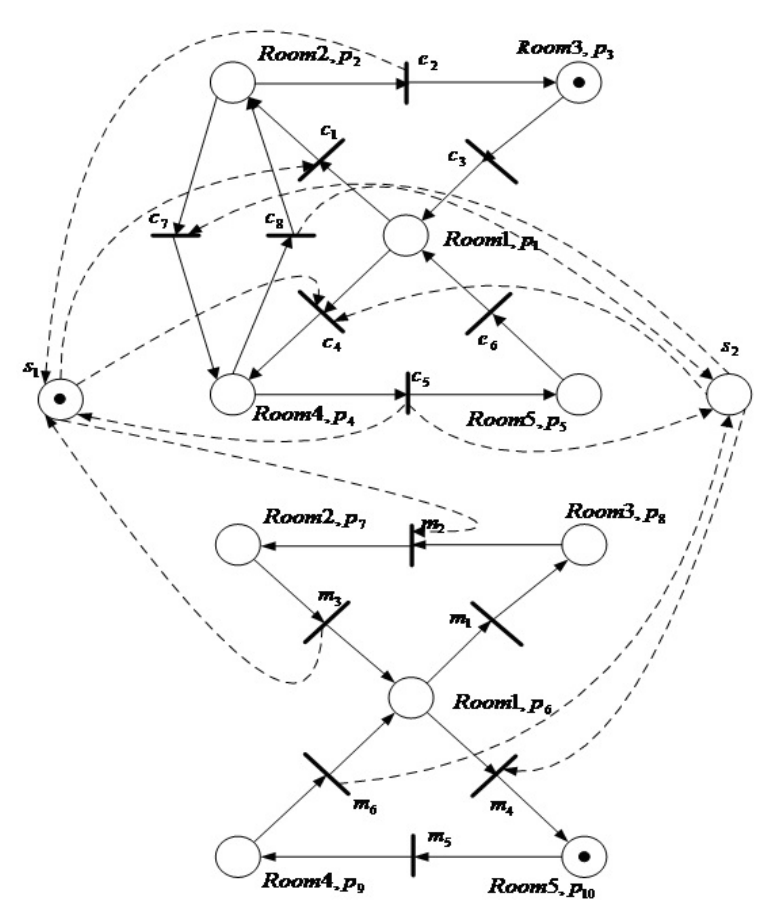

$$
M^{\prime}=\left[\begin{array}{ccccccc}
1 & 0 & 0 & 1 & 0 & 0 & 0 \\
-1 & 0 & 0 & 0 & 1 & 0 & 0 \\
0 & 1 & 0 & 0 & 0 & 1 & 0 \\
0 & -1 & 1 & 0 & 0 & 0 & 1 \\
0 & 0 & -1 & 0 & 1 & 0 & 0 \\
-1 & 0 & 0 & 0 & 0 & 0 & 1
\end{array}\right]
$$

Figure 9 The controlled Petri net model of case 2

This is:

$$
\begin{gathered}
R=\left[\begin{array}{llll}
0 & 1 & 0 & 0 \\
0 & 0 & 0 & 1
\end{array}\right] \\
C^{\prime}=R D+C \\
=\left[\begin{array}{ccccccccccc}
1 & -1 & 1 & -1 & 0 & 0 & 1 & -1 & 0 & 0 & 0 \\
0 & 0 & 1 & -1 & 1 & -1 & 0 & 0 & 1 & 0 & -1
\end{array}\right]
\end{gathered}
$$

That is, inadmissible constraints (17) and (19) are transformed into new admissible constraints:

$$
\begin{aligned}
& \left(v_{c 1}-v_{c 2}+v_{c 4}-v_{c 5}\right)+\left(v_{m 2}-v_{m 3}\right) \leq 1 \\
& \left(v_{c 4}-v_{c 5}+v_{c 7}-v_{c 8}\right)+\left(v_{m 4}-v_{m 6}\right) \leq 1
\end{aligned}
$$

Design the controllers according to inequalities (21) and (22). The controlled Petri net is shown in Figure 8 , where the broken line parts are controllers $s 1$ and $s 2$. If take the method of [9] to design the controller, then the dimension of matrix $M$ is $12 \times 13$, however the dimension of matrix $M$ in this paper is $6 \times 7$. The method cuts down the calculating works during controller designing through reducing the matrix dimension, because it only considers the places related to uncontrollable transitions and the transitions related to these places.

\section{Conclusion}

In this paper, a supervisory control approach has been proposed for Petri net plants with uncontrollable transitions that enforce the conjunction of a set of linear inequality constraints on the Parikh vector or place 
markings. The proposed method is entirely straightforward logically, graphically, technologically, and able to avoid deadlock. This method becomes a useful alternative to mapping monitors when there does not exist an admissible constraint after the constraint transformation for the given problem. The method is based on part net design, and it only considers the direct or indirect transitions related to the constraints. So the computation required to find the Petri net controller is quite simple. Compared with the synthesis method proposed by Wang [8] and Moody [4], the method should be widely applicable and of practical interest. The efficiency of the method has been shown by means of examples. The method with these advantages lends itself as a practical approach to control synthesis of large and complex DES.

\section{References}

[1] Huang Y S, "Design of deadlock prevention supervisors using Petri nets", The International Journal of Advanced Manufacturing Technology, No.3, pp.3493625, 2007.

[2] Uzam M, and Wonham W M, "A hybrid approach to supervisory control of discrete event systems coupling RWsupervisors to Petri nets", The International Journal of Advanced Manufacturing Technology, No.28, pp.747-760, 2006.

[3] Uzam M, "Synthesis feedback control elements for discrete event systems using Petri net models and theory of regions", The International Journal of Advanced Manufacturing Technology, Vol.24, pp.4869, 2004.

[4] Moody J O, and Antsaklis P J, "Petri Net Supervisors for DES with Uncontrollable and Unobservable Transitions", IEEE Transactions on Automatic Control, Vol.45, No.3, pp.462-476, 2000.

[5] M. Uzam, "On suboptimal supervisory control of Petri nets in the presence of uncontrollable transitions via monitor places". The International Journal of Advanced Manufacturing Technology, Vol.47, No.5, pp.567-579, 2009.

[6] Iordache M V, Moody J O, and Antsaklis P J, "Synthesis of Deadlock Prevention Supervisors Using Petri Nets", IEEE Transactions on Robotics and Automation, Vol.18, No.1, pp.59-68, 2002.

[7] Zhong C F, Li Z W, "A deadlock prevention approach for flexible manufacturing systems without complete siphon enumeration of their Petri net models". Engineering with Computers, Vol.25, pp.269-278, 2009.

[8] Wang S G, and Yang G F, "A Method for the Design of Petri Net Controller Enforcing General Linear Constraints", Journal of Software, Vol.16, No.3, pp.419-426, 2005.
[9] Zhang Y Y, Supervisor synthesis for discrete event system based on constraint transformation, Zhejiang university, 2007.

[10] Tao Z, Xie L Y, Liang D, "Controller design of DES Petri nets with mixed constraint", Chinese journal of aeronautics, Vol.18, No.3, pp.283-288, 2005.

[11] Moody J O, “Antsaklis P J, Automated Design of A Petri Net Feedback Control for a Robotic Assembly Cell", Emerging Technologies and Factory Automation, Vol.45, No.2, pp.117-128, 1995.

[12] O.Boissel, Optimal feedback control design for discrete-event process systems using simulated annealing, Notre Dame, University of Notre Dame, 1993.

[13] J.O. Moody, P. J. Antsaklis, "Charactrization of feasible controls for Petri nets with unobservable transitions". In proc. IEEE of the 1997 American Control Conference, New Orieans, 1997, 2354-2358. 